\title{
Role of deregulated microRNAs in non-small cell lung cancer progression using fresh-frozen and formalin-fixed, paraffin-embedded samples
}

\author{
YAHONG WANG $^{1 *}$, JIE CHEN $^{2 *}$, ZIYING LIN $^{1 *}$, JUN CAO $^{3}$, HAILI HUANG $^{1}$, \\ YUN JIANG ${ }^{1}$, HUIJUAN HE ${ }^{1}$, LAWEI YANG ${ }^{1}$, NINA REN $^{1}$ and GANG LIU ${ }^{1 *}$ \\ ${ }^{1}$ Clinical Research Center; ${ }^{2}$ Department of Cardiothoracic Surgery; \\ ${ }^{3}$ Pathological Diagnosis and Research Center, Affiliated Hospital of Guangdong Medical University, \\ Zhanjiang, Guangdong 524001, P.R. China
}

Received July 14, 2014; Accepted March 2, 2015

DOI: $10.3892 / \mathrm{ol} .2015 .3976$

\begin{abstract}
Non-small cell lung cancer (NSCLC) is responsible for the highest number of cancer-associated mortalities worldwide, and the five-year survival rate is $<15 \%$ following the initial diagnosis. MicroRNAs (miRNAs) serve important functions in a number of human diseases, including cancer. The present study investigated the expression status, clinical relevance and functional role of miRNA in NSCLC. miRNA expression profiling was performed in lung adenocarcinoma and adjacent unaffected lung tissues using 47 groups of fresh-frozen (FF) and 45 of formalin-fixed, paraffin-embedded (FFPE) samples from 11 pulmonary bulla. miR-21, $-30 \mathrm{e},-363$ and -623 were further examined for differential expression in two independent cohorts. Other miRNAs, including miR-5100 and miR-650, were upregulated, while miR-10a and -26b were downregulated in FF NSCLC tissues. The associations between these miRNAs and their clinicopathological features were also investigated. miR-363, -10a and -145 were associated with lymph node status $(\mathrm{P}=0.002,0.005$ and 0.007 , respectively) and miR-650 and -145 were associated with differentiation $(\mathrm{P}=0.01$ and 0.05 , respectively). No associations were identified for the other miRNAs examined. In the FFPE NSCLC samples,
\end{abstract}

Correspondence to: Dr Gang Liu, Clinical Research Center, Affiliated Hospital of Guangdong Medical University, 57 Renming Avenue, Zhanjiang, Guangdong 524001, P.R. China

E-mail: gangliu11@gdmc.edu.cn

"Contributed equally

Abbreviations: NSCLC, non-small cell lung cancer; FF, fresh frozen; FFPE, formalin-fixed, paraffin-embedded

Key words: non-small cell lung cancer, microRNA, reverse transcription-quantitative polymerase chain reaction, biomarker
miR-30e-5p correlated with the differentiation of the tissue $(\mathrm{P}=0.011)$. The present study indicates that these miRNAs may be appropriate candidates for molecular diagnostic and prognostic markers in NSCLC.

\section{Introduction}

Lung cancer is the leading cause of cancer-associated mortality worldwide, and the majority of broncogenic carcinomas are cases of non-small cell lung cancer (NSCLC) (1). NSCLC can be histologically subdivided into four major subtypes with distinct pathological characteristics as follows: i) Adenocarcinoma; ii) squamous cell carcinoma; iii) large cell carcinoma; and iv) 'other', including neuroendocrine cancer and carcinoids (2). NSCLC is often diagnosed at the advanced stages of disease progression, resulting in a poor prognosis and an overall five-year survival rate of $\sim 14 \%$. However, the five-year survival rate in patients with stage I NSCLC that has been resected can be as high as $83 \%(2,3)$. Despite improvements in early diagnosis and treatment responses, the overall five-year survival rates for NSCLC patients remains low (15\%) and the recurrence rate is high, even in cases with early diagnosis (4). The identification of biomarkers for lung cancer may aid early diagnosis and accurate prognosis. Therefore, it is important to identify novel and reliable biomarkers for molecular targeted therapy.

Advances in genomic technologies have generated numerous candidate biomarkers with potential clinical value in NSCLC. A complementary approach to performing gene expression profiling in NSCLC is to analyze microRNA (miRNA) expression signatures. miRNAs are a class of short RNAs that are 19-25 nucleotides in length and regulate gene expression by binding to sequences in the 3'-untranslated region (3'UTR) of an expressed mRNA. This results in modulation of translation efficiency or degradation of the targeted mRNA (5). miRNAs have been associated with cell signaling pathways involved in cell differentiation, proliferation and survival. The aberrant expression of miRNAs has been demonstrated in a number of different types of tumor (6), including lung cancer (7). miRNA profiling has 
been proposed as a strategy for classifying NSCLCs, and appears to be reliable in certain cases (8-10).

Increasing evidence suggests that miRNAs are expressed aberrantly in numerous types of human cancer and that they serve a significant role in carcinogenesis and cancer progression (11). Aberrant expression of miR-21 has been associated with several types of cancer, and a previous study demonstrated that it is overexpressed in tumor tissues of patients with advanced-stage NSCLC. In addition, the miR-12 target PTEN, which is a negative regulator of the PKB-AKT pathway, is involved in NSCLC cell line invasion (12-14). A previous study demonstrated that miR-125a-3p and $-5 p$ are downregulated in primary NSCLC tumors, but that they serve an opposing role in lymph node metastases (15). miR-328 and miR-206 have also been associated with NSCLC brain metastasis $(16,17)$. Arora et al (16) reported that miR-155 is overexpressed in adenocarcinoma, and further studies have demonstrated miR-155 overexpression in squamous cell lung cancer $(18,19)$.

Differential expression of miRNAs has been observed in previous studies of lung cancer and adjacent normal tissues $(18,20)$. In order to identify novel biomarkers of lung cancer, a number of investigators have performed miRNA expression profiling studies in cell lines, tissue samples or serum samples (21-23). In the present study, the expression levels of 15 miRNAs were examined using reverse transcription-quantitative polymerase chain reaction (RT-qPCR) in NSCLC and adjacent normal tissues. The expression levels of the 15 miRNAs were also determined by RT-qPCR in formalin-fixed, paraffin-embedded (FFPE) tissue from NSCLC and non-carcinomatous (pulmonary bulla) tissues. The aim of the present study was to identify possible candidate miRNAs to be used as biomarkers for NSCLC.

\section{Materials and methods}

Ethical statement. All samples were obtained from the Affiliated Hospital of Guangdong Medical University (Zhanjiang, China). The study protocol was approved by the institutional review boards of participating centers (Affiliated Hospital of Guangdong University). Written informed consent was obtained from all patients.

Samples. Tissues were obtained from 47 patients with NSCLC diagnosed between January 2013 and March 2014. The samples were as follows: Fresh-frozen (FF) tissues resected from the 47 patients $(n=140,47$ NSCLC samples, 46 samples of tissues adjacent to the carcinoma and 47 samples of normal lung tissues) and 56 samples from FFPE tissues ( $n=56$, including 45 carcinomatous NSCLC tissues and 11 non-carcinomatous pulmonary bulla samples). The samples were analyzed histologically to assess the level of tumor component (a minimum of $80 \%$ tumor cells) and the quality of the harvested material. Paracarcinomatous tissues adjacent to the tumor (distance from the primary tumor $<2 \mathrm{~cm}$ ) and normal tissues (distance from the primary tumor $>5 \mathrm{~cm}$ ) were defined histologically using classical pathological approaches (24).

Clinical and pathological data were obtained from the medical records and centrally reviewed for the purpose of the study. Clinicopathological information [smoking, age, gender, pathologic subtype, tumor node metastasis classification, tumor stage, lymph node stage, differentiation status and adjuvant therapy following surgery] from all patients that participated in the present study is summarized in Tables I and II.

RNA extraction. The total RNA was extracted using TRIzol reagent (Invitrogen Life Technologies, Carlsbad, CA, USA) and reverse transcribed using a miRcute miRNA First-strand cDNA Synthesis kit (Tiangen Biotech Co., Ltd., Beijing, China). Unstained FFPE tissues were sectioned at $10-\mu \mathrm{m}$ thickness and 6 tissue sections were placed into a $1.5-\mathrm{ml}$ tube. The sections were deparaffinized by adding $1 \mathrm{ml}$ xylene (Guangzhou Chemical Reagent Factory, Guangzhou, China) and the sections were vortexed vigorously at room temperature for 3 min. Following washes in $70 \%$ and absolute ethanol to remove xylene, the RNA was extracted with the miRNeasy FFPE kit (Qiagen China Co., Ltd., Shanghai, China). Following extraction, all RNA samples were stored at $-80^{\circ} \mathrm{C}$ until use.

Quantitative polymerase chain reaction ( $q P C R)$. All miRNA expression levels were quantified via qPCR using primers designed by Tiangen Biotech Co., Ltd., an miRcute miRNA qPCR Detection kit (SYBR Green) (Tiangen Biotech Co., Ltd.) and a Rotor-Gene Q 2plex HRM System (Qiagen China Co., Ltd.). All the samples were normalized against the endogenous control snRNA U6 and fold changes were calculated relative to the control. The relative expression levels were calculated using the $2^{-\Delta \Delta \mathrm{Cq}}$ method.

Study selection. The online literature database PubMed was used to identify lung cancer miRNA expression profiling studies published from January 2003 until May 2014 (last accessed on 15 May 2014), by means of the 'Medical Subject Heading' terms: 'NSCLC' and 'microRNAs' in combination with the keywords 'profiling' and 'humans'. The results of previous studies to identify circulating miRNAs specific to lung cancer were inconsistent (24-27), and potential biomarkers remain to be elucidated and exploited. Based on these previous findings, a list of 15 miRNAs was compiled, whose expression has already been reported as deregulated in cancer, or has not been investigated (Table III).

Statistical analysis. SPSS software, version 17.0 (SPSS, Inc., Chicago, IL, USA) was used for statistical analysis. The Wilcoxon test was used to examine the differences of miRNA expression between lung carcinoma and normal subjects; a Spearman's Rho test was performed to compare the relative expression of miRNA. Pearson's Correlation Coefficient was used to analyze the association between the expression of miRNAs and clinicopathological features of the patients. Experimental data were expressed as the mean \pm standard error. To compare the means of two groups, a two-tailed Student's t-test was used and $\mathrm{P}<0.05$ was considered to indicate a statistically significant difference.

\section{Results}

MicroRNA signatures can efficiently distinguish NSCLC tissues from adjacent normal tissues. A meta-analysis of human lung cancer microRNA expression profiling studies 


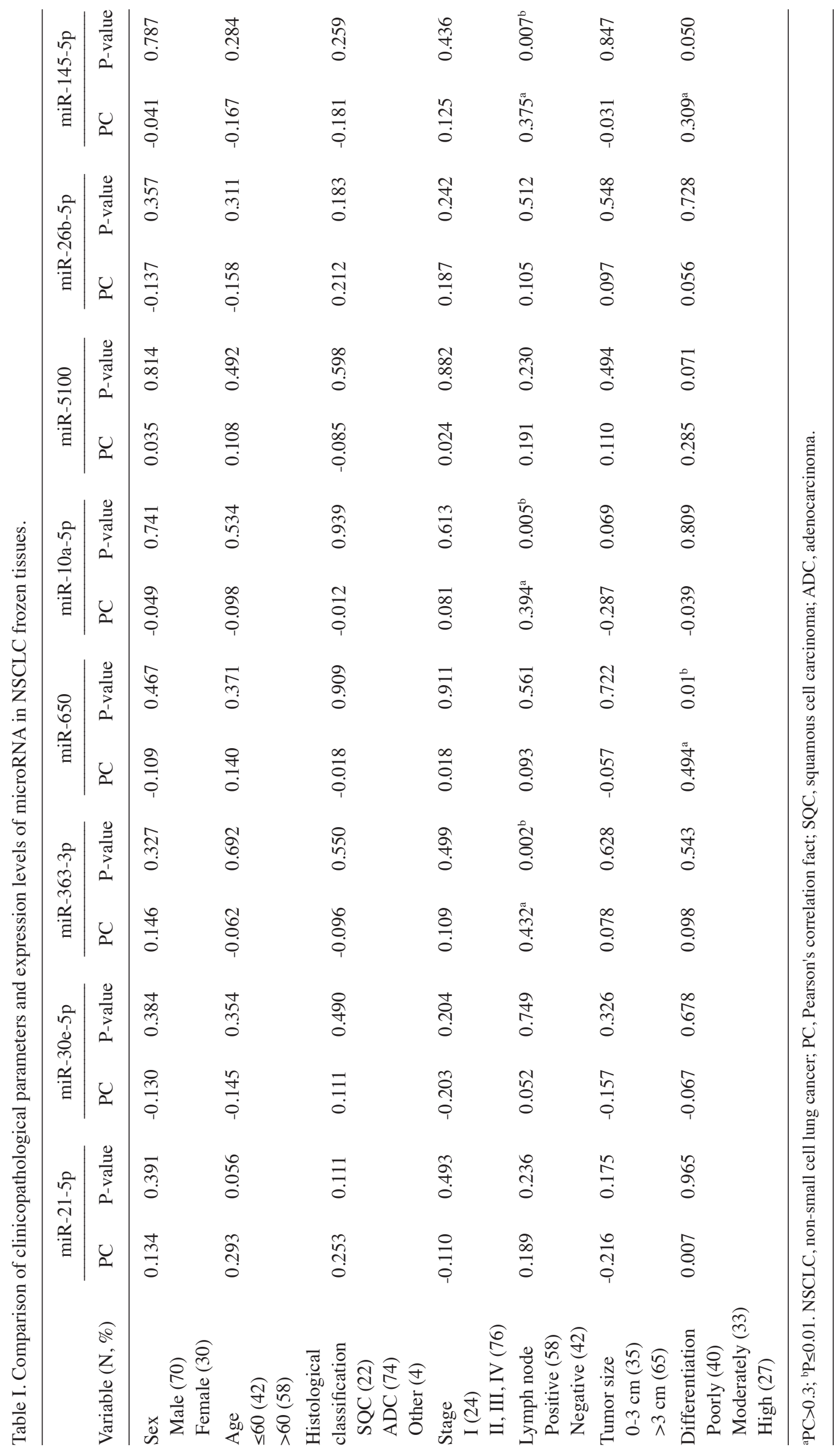


Table II. Association between the expression of four miRNAs in FFPE samples and the clinicopathological characteristics of the tumor from 45 NSCLC patients.

\begin{tabular}{|c|c|c|c|c|c|c|c|c|}
\hline \multirow[b]{2}{*}{ Variable (N, \%) } & \multicolumn{2}{|c|}{$\operatorname{miR}-21-5 p$} & \multicolumn{2}{|c|}{$\operatorname{miR}-30 e-5 p$} & \multicolumn{2}{|c|}{ miR-363-3p } & \multicolumn{2}{|c|}{$\operatorname{miR}-623$} \\
\hline & $\mathrm{PC}$ & P-value & $\mathrm{PC}$ & P-value & $\mathrm{PC}$ & P-value & $\mathrm{PC}$ & P-value \\
\hline $\begin{array}{l}\text { Sex } \\
\text { Male (73) } \\
\text { Female (26) }\end{array}$ & -0.079 & 0.605 & 0.037 & 0.808 & 0.098 & 0.520 & 0.098 & 0.520 \\
\hline $\begin{array}{l}\text { Age } \\
\leq 60(26) \\
>60(63)\end{array}$ & -0.131 & 0.388 & 0.048 & 0.754 & 0.127 & 0.407 & 0.068 & 0.655 \\
\hline $\begin{array}{l}\text { Histological } \\
\text { classification } \\
\text { SQC (46) } \\
\text { ADC (54) } \\
\text { Other (0) }\end{array}$ & -0.075 & 0.625 & 0.125 & 0.413 & -0.150 & 0.327 & -0.087 & 0.570 \\
\hline $\begin{array}{l}\text { Stage } \\
\text { I (44) } \\
\text { II, III, IV (56) }\end{array}$ & 0.150 & 0.326 & -0.115 & 0.451 & 0.146 & 0.338 & 0.033 & 0.828 \\
\hline $\begin{array}{l}\text { Lymph node } \\
\text { Positive (36) } \\
\text { Negative (64) }\end{array}$ & 0.066 & 0.664 & -0.084 & 0.584 & 0.216 & 0.154 & -0.077 & 0.614 \\
\hline $\begin{array}{l}\text { Tumor size } \\
0-3 \mathrm{~cm}(60) \\
>3 \mathrm{~cm}(40)\end{array}$ & -0.003 & 0.987 & -0.124 & 0.419 & 0.177 & 0.224 & 0.079 & 0.608 \\
\hline $\begin{array}{l}\text { Differentiation } \\
\text { Poorly (36) } \\
\text { Moderately (40) } \\
\text { High (24) }\end{array}$ & 0.274 & 0.069 & $0.376^{\mathrm{a}}$ & $0.011^{\mathrm{b}}$ & 0.203 & 0.180 & 0.013 & 0.930 \\
\hline
\end{tabular}

NSCLC, non-small cell lung cancer; $\mathrm{PC}$, Pearson's correlation fact; $\mathrm{SQC}$, squamous cell carcinoma; $\mathrm{ADC}$, adenocarcinoma. ${ }^{\mathrm{a}} \mathrm{PC}>0.3$; ${ }^{\mathrm{b}} \mathrm{P}<0.05$.

Table III. List of miRNA probes used to profile tissue samples from non-small cell lung cancer.

\begin{tabular}{lcccc}
\hline Hsa-miR-30e-5p & Hsa-miR-10a-5p & Hsa-miR-21-5p & Hsa-miR-26b-5p & Hsa-miR-17-3p \\
Hsa-miR-211-3p & Hsa-miR-1246 & Hsa-miR-5100 & Hsa-miR-181a-5p & Hsa-miR-363-3p \\
Hsa-miR-650 & Hsa-miR-623 & Hsa-miR-145-5p & Hsa-miR-155-5p & Hsa-miR-125b-5p \\
\hline
\end{tabular}

$(18,21,28,29)$ was performed, which identified 15 miRNA genes of possible interest. The initial investigation sought to determine whether the expression of any of the 15 miRNAs was able to identify malignant NSCLC tissues in Chinese patients. Primary NSCLC lung cancer tissues (T), paracarcinomatous tissues $(\mathrm{P})$ and their corresponding adjacent normal lung tissues ( $\mathrm{N} ; \geq 5 \mathrm{~cm}$ from the tumor) in 47 patients were examined using microRNA-specific reverse transcription-qPCR (RT-qPCR). The relative expression level of the following microRNAs significantly differed between $\mathrm{T}, \mathrm{P}$ and $\mathrm{N}$ tissues: miR-21-5p, -10a-5p, -26b-5p, -363-3p, -5100, $-623,-650,-145-5 p$ and $-30 e-5 p$ (Fig. 1). miR-21-5p was upregulated in $32 / 47$ NSCLC cases $(68.1 \%, \mathrm{P}<0.001)$. By comparing NSCLC with normal lung tissues, NSCLC with paratumor tissues and paratumor with normal lung tissues, 9 miRNAs were identified with significantly different expression levels (Table IV).

Validation of the selected miRNAs expression in FFPE tissue. It was hypothesized that FFPE tissue storage may improve the stability of mRNA (30). To determine if the identified miRNAs could be reliably detected in the specimens, and whether the previously identified miRNAs (miR-21-5p, $-10 a-5 p,-26 b-5 p,-363-3 p,-5100,-650,-145-5 p,-30 e-5 p$ and miR-623) were differentially expressed in FFPE samples from NSCLC compared with those from pulmonary bullae, their expression levels were quantified by RT-qPCR. Each of the 15 miRNAs presented $\mathrm{Ct}$ values $\leq 40$, indicating that the 
Table IV. Differential expression levels of miRNA in tumor, paratumor and adjacent normal lung tissue.

\begin{tabular}{lccc}
\hline miRNA & T vs. N (P-value) & T vs. P(P-value) & P vs. N (P-value) \\
\hline miR-21-5p & $<0.001$ & $>0.05$ & $<0.05$ \\
miR-650 & $<0.001$ & $>0.05$ & $<0.05$ \\
miR-5100 & $<0.001$ & $<0.05$ & $<0.05$ \\
miR-363-3p & $<0.001$ & $<0.05$ & $>0.05$ \\
miR-26b-5p & $<0.001$ & $<0.05$ & $>0.05$ \\
miR-623 & $>0.05$ & $>0.05$ & $<0.05$ \\
miR-145-5p & $<0.001$ & $<0.001$ & $>0.05$ \\
miR-10a-5p & $<0.001$ & $<0.001$ & $>0.05$ \\
miR-30e-5p & $<0.001$ & $<0.001$ & $>0.05$ \\
\hline
\end{tabular}

T, tumor; $\mathrm{N}$, adjacent normal lung tissue; $\mathrm{P}$, paratumor.

A

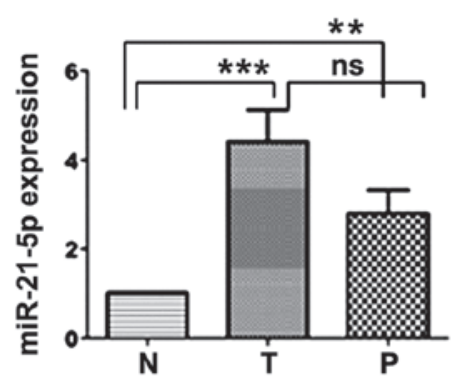

D

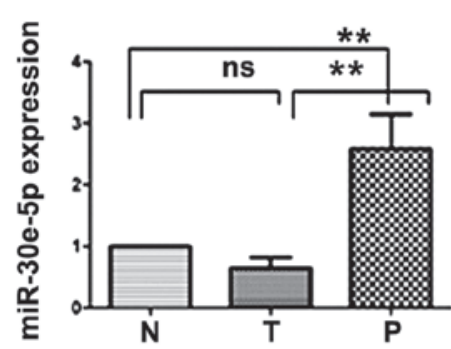

G

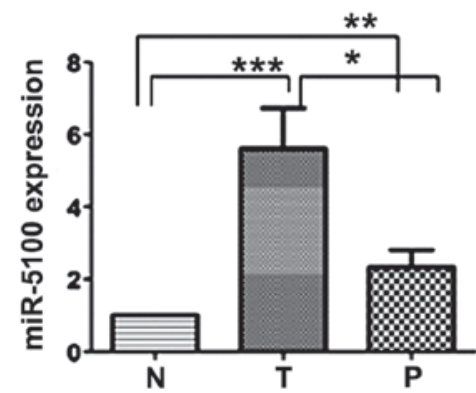

B

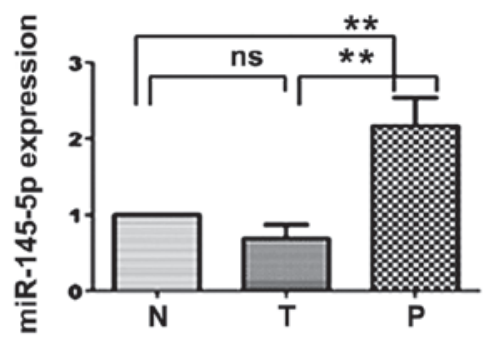

E

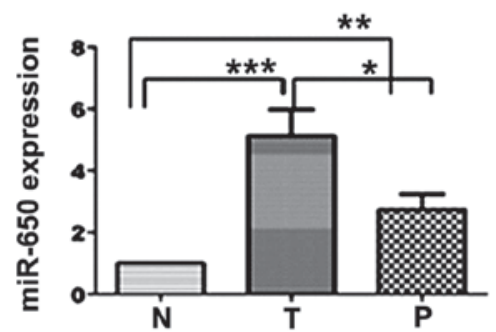

H

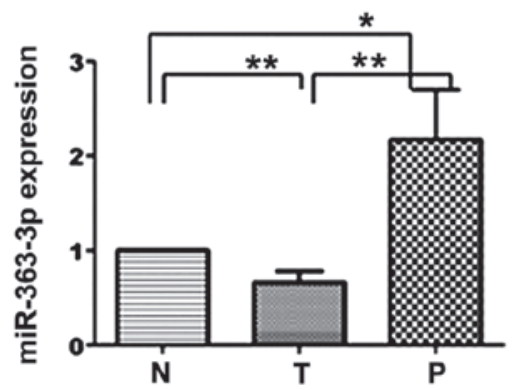

C

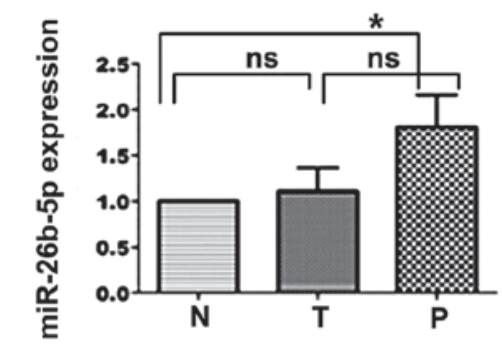

F

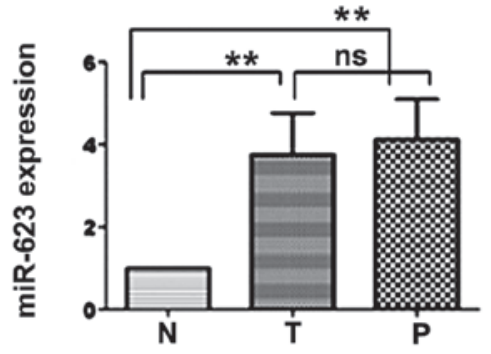

I

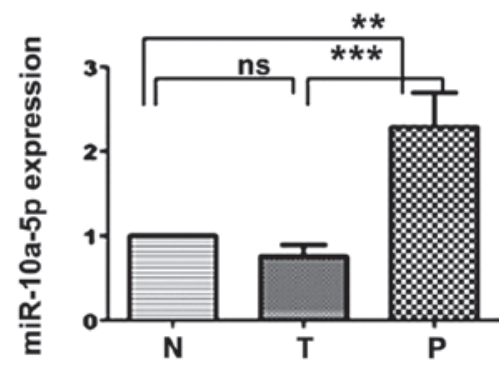

Figure 1.RT-qPCR analysis of deregulated microRNA expression in NSCLC frozen tissues. Three groups of comparisons were performed: i) Tumor vs. paratumor; ii) tumor vs. adjacent normal lung tissues; and iii) paratumor vs. adjacent normal lung tissues. Relative expression of (A) hsa-miR-21-5p, (B) hsa-miR-145-5p, (C) hsa-miR-26b-5p, (D) hsa-miR-30e-5p, (E) hsa-miR-650, (F) hsa-miR-623, (G) hsa-miR-5100, (H) hsa-miR-363-3p and (I) hsa-miR-10a-5p were determined in reference to an internal U6 snRNA control. Relative expression values are presented as the normalized mean \pm standard error. Data derived from RT-qPCR are presented as $2^{-\Delta \Delta C q}$ values. ${ }^{*} \mathrm{P}<0.05$ vs. $\mathrm{N} .{ }^{* * *} \mathrm{P}<0.01$ vs. $\mathrm{N}$. ${ }^{* * * *} \mathrm{P}<0.001$ vs. N. NSCLC, non-small cell lung cancer; RT-qPCR, reverse transcription-quantitative polymerase chain reaction; $\mathrm{N}$, adjacent normal lung tissue; $\mathrm{T}$, tumor; $\mathrm{P}$, paratumor; ns, not significant.

miRNAs could easily be measured in these FFPE samples (data not shown). In addition, the median $2^{-\Delta \Delta \mathrm{Cq}}$ levels of miR-623, -21-5p, -30e-5p and $-363-3 p$ were significantly different between NSCLC and pulmonary bulla patient samples (Fig. 2), whereas the observed level of miR-26b-5p, $-5100,-650$ and $-145-5 p$ did not differ significantly between NSCLC and pulmonary bulla patients (Fig. 2).
Association between clinicopathological characteristics and differentially expressed miRNAs in NSCLC. The association between the differentially expressed miRNAs and the clinicopathological features (such as clinical stage, lymph node status, tumor size, histological classification and differentiation) of the associated tumors, was analyzed. miR-363, -10a and -145 were associated with lymph node 
A

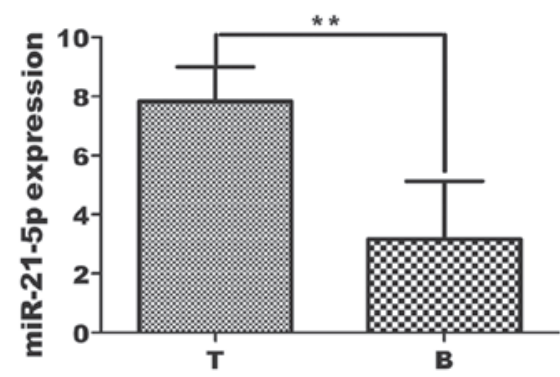

C

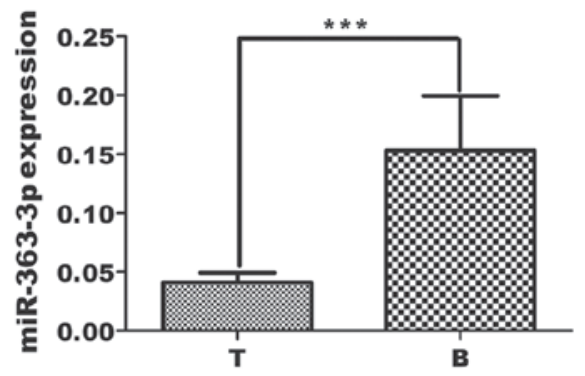

B

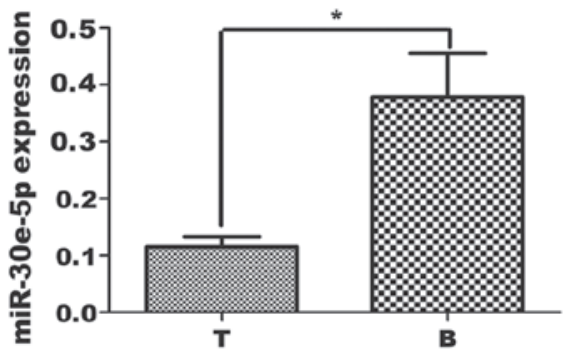

D

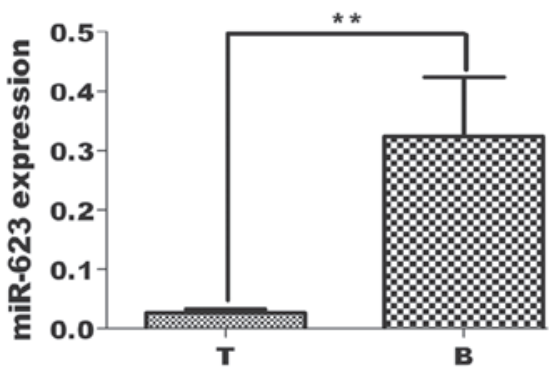

Figure 2. RT-qPCR analysis of deregulated microRNA expression in NSCLC FFPE tissues. One comparison was performed: Tumor vs. pulmonary bulla. Relative expression of (A) hsa-miR-21-5p, (B) hsa-miR-30e-5p, (C) hsa-miR-363-3p and (D) hsa-miR-623 were determined in reference to an internal U6 snRNA control. Data derived from RT-qPCR are presented as $2^{-\Delta \Delta \mathrm{Cq}}$ values and the relative expression values are presented as the normalized mean \pm standard error. ${ }^{*} \mathrm{P}<0.05,{ }^{* *} \mathrm{P}<0.01$ and ${ }^{* * *} \mathrm{P}<0.001$. RT-qPCR, reverse transcription-quantitative polymerase chain reaction; FFPE, formalin-fixed, paraffin-embedded; NSCLC, non-small cell lung cancer; $\mathrm{T}$, tumor; B, pulmonary bulla.

status $(\mathrm{P}=0.002,0.005$ and 0.007 , respectively) and miR-650 and -145 were associated with differentiation $(\mathrm{P}=0.01$ and 0.05 , respectively; Table I). No association was identified for the other miRNAs examined. In the FFPE NSCLC samples, miR-30e-5p correlated with the differentiation of the tissue $(\mathrm{P}=0.011)$ in these patient samples (Table II).

\section{Discussion}

The critical role of miRNAs in cancer has become increasingly apparent. Previous studies have demonstrated that these small regulatory RNA molecules participate in a diverse set of cell signaling processes, including apoptosis, cell proliferation and epithelial-to-mesenchymal transition $(12,21,31,32)$. In the present study, miRNA expression levels in NSCLC tissues and their corresponding normal lung tissues were quantified using RT-qPCR analysis. The present study identified 8 miRNAs that were significantly differentially expressed in the NSCLC tissues compared with the corresponding normal specimens, of which 4 miRNAs were upregulated and 4 miRNAs were downregulated. The data presented in the current study are in agreement with the results of previously published miRNA profiling results (18). To the best of our knowledge, the present study is the first to report the differential expression of miR-5100 in cancerous tissues and is the first to report altered miR-363-3p expression in lung cancer. The expression level of miR-145 was associated with the differentiation and lymph node status $(\mathrm{P}=0.05$ and 0.007 , respectively) and mir-650 was associated with the differentiation status $(\mathrm{P}=0.01)$.
A previous study reported that hsa-miR-145 expression levels are reduced in colon cancer (33), which was also reflected in NSCLC tissues in the present study. In addition, the present study also demonstrated that hsa-miR-145 expression levels were associated with lymph node status $(\mathrm{P}=0.007)$. Upregulation of hsa-miR-21 expression levels has been observed in a number of human cancer specimens and it has been demonstrated that hsa-miR-21 targets and downregulates the tumor suppressor gene, tumor suppressor tropomyosin 1 (34). In the present study, compared with normal lung tissue, the expression level of miR-21-5p was observed to be increased in the paratumor tissues, and further increased in the NSCLC tissues (Fig. 1A), suggesting that increased miR-21-5p expression may also be involved in the development and progression of lung cancer.

A previous study identified a 5-miRNA signature (hsa-miR-155, hsa-miR-17-3p, hsa-let-7a-2, hsa-miR-145 and hsa-miR-21) whose expression was significantly increased in lung squamous cell carcinoma tissue samples compared with normal lung tissues, and it correlated with the prognosis and survival outcome of the patients (35). However, this 5-miRNA signature did not significantly predict patient outcome in the data set from the present study, in which miR-17 and miR-155 expression levels were not significantly altered in the NSCLC tissue.

A previous study reported that miR-181a expression is upregulated in papillary thyroid carcinoma (36), while others have reported it to be downregulated $(37,38)$. No changes in miR-181a expression levels were observed in the NSCLC tissues in the present study, in contrast to Gao et al (38), who observed low expression levels of miR-181a in tissues from 
NSCLC patients. This difference may be due to the different tissue processing methods and the sample origin.

The present study also identified that miR-30e-5p is significantly downregulated in NSCLC tissues, by comparing FFPE non-cancerous adjacent tissues and NSCLC tissues. This result is consistent with Markou et al (24) who also observed downregulation of miR-30e-5p in an independent group of 40 matched fresh-frozen tissues, 37 plasma samples from NSCLC patients and 28 healthy donors. Consistent with a previous study (39), the present study demonstrated that miR-30e-5p expression was significantly upregulated in paratumor tissue compared with adjacent lung tissues or tumor tissues, however, no significant differences were identified between miR-30e-5p expression in tumor and adjacent normal lung tissue. Furthermore, miR-30e-5p expression was significantly associated with the tissue differentiation in FFPE samples (Fig. 1D).

In the present study, miR-650 expression levels were significantly higher in NSCLC tissues than in adjacent non-cancerous tissues (Fig. 1E) and associated with differentiation (Table I). Similarly, Huang et al (40) previously demonstrated that miR-650 expression levels were upregulated in human lung adenocarcinoma tissues and correlated with advanced clinical stage and a higher incidence of lymph node metastasis (40). However, in the FFPE samples examined in the present study, no significant change in miR-650 expression level was observed, which may indicate that the stability of mRNA is an issue with regard to extraction from FFPE tissues is necessary (data not shown).

The downregulation of miR-623 expression levels in malignantly transformed oral leukoplakia has previously been observed (41). However, in the present study, miR-623 was significantly upregulated in tumor tissues compared with adjacent normal tissue $(\mathrm{P}<0.01$; Fig. $1 \mathrm{~F})$. Overexpression of miR-623 has also been observed in esophageal cancer tissues compared with tissues adjacent to the tumors (24).

There have been few studies investigating miR-363 expression levels in different types of human cancer. To the best of our knowledge, the present study reports for the first time that miR-363 is downregulated in NSCLC tissues compared with the non-cancerous adjacent tissues (Fig. 1H). miR-363 has been proposed as a novel biomarker for the diagnosis of colorectal cancer $(42,43)$, and it is downregulated in head and neck squamous cell carcinoma tissues with lymph node metastasis (44).

Furthermore, the present study demonstrated for the first time that miR-5100 is upregulated in NSCLC compared with its corresponding adjacent normal tissue. Fig. 1G indicates that, compared with normal lung tissues, the expression level of miR-5100 is increased in paratumor tissues, and further increased in tumor tissues; a gradual increase was observed.

A previous study demonstrated that miR-10a-5p expression levels were significantly different in acute myeloid leukemia compared with control serum samples (45). The results of the present study demonstrated that miR-10a-5p expression levels in NSCLC tissues were downregulated compared with normal tissues (Fig. 1I), and that they were associated with lymph node status $(\mathrm{P}=0.005)$.

Previous studies have indicated that RT-qPCR can be performed to quantify miRNA expression using archival
FFPE tissue samples $(46,47)$. The present study employed FFPE tissue samples from lung cancer and normal tissues to identify miRNAs deregulated in patients with lung cancer: miRNA-21-5p was upregulated and miR-623, $-30 e-5 p$ and $-363-3 p$ were downregulated in lung cancer samples compared with control pulmonary bulla specimens. In addition, the expression level of miRNA-30e-5p was associated with the differentiation status of the tumor $(\mathrm{P}=0.011$; Table II). However, no alteration in the expression levels of miR-145-5p, -26b-5p, -650, -5100 and -10a-5p was detected in FFPE tissue samples compared with normal pulmonary bulla tissues. One possible explanation is that the formalin cross-links that form between proteins and nucleic acids in the tissue complicates the quantification of the miRNAs (48). Alternatively, the RNA is significantly damaged prior to, during and following the FFPE procedure and is degraded into fragments $<200$ base pairs in length. Thus, the efficiency of RNA extraction and RT are reduced and as a result, the quantification of RNA extracted from FFPE tissues using PCR-based methods is less reliable than in frozen tissues (49).

The following limitations of the present study should be taken into consideration: i) The sample size is relatively small. A larger scale study to confirm these data is required; ii) the precise mechanisms by which these miRNAs and their target mRNAs regulate NSCLC progression remain largely unclear and further mechanistic and external validation through in vitro and in vivo studies are required to determine the clinical significance of miRNA expression and their role in the development of NSCLC; and iii) the current study is retrospective, with limited scope for generalizability as all the patients were from China, and the distribution of clinical characteristics may be different in patients from other countries of other ethnicities.

In conclusion, the present study demonstrated aberrant expression of 8 miRNAs in tissues from patients with NSCLC. miR-650 and miR-10a were aberrantly expressed, miR-145 was associated with lymph node metastasis; and miR-650 was associated with differentiation. The present study demonstrates for the first time that miR-5100 is upregulated and that miR-363 is downregulated in NSCLC and associated with lymph node metastasis. The expression levels of miR-26b-5p, miR-1246, miR-211-3p and miR-125b-5p were unchanged in the NSCLC tissues, in contrast with previous reports that observed upregulation or downregulation of these miRNAs in melanoma, multiple myeloma or hepatitis B virus-related hepatocellular carcinoma $(47,50,51)$. The present study provides further experimental data for these candidate miRNAs in order to aid the identification of reliable molecular diagnostic and prognostic markers of NSCLC in the future.

\section{Acknowledgements}

The present study was supported by the Science \& Technology Innovation Fund of Guangdong Medical University (grant no. STIF201109); the National Natural Science Foundation of China project (grant nos. NSFC81172615 and NSFC81570062) and the Guangdong Medical University Science Fund (grant nos. M2014046 and M2014032). 


\section{References}

1. Siegel R, Ma J, Zou Z and Jemal A: Cancer statistics, 2014. CA Cancer J Clin 64: 9-29, 2014.

2. Minna JD, Roth JA and Gazdar AF: Focus on lung cancer. Cancer Cell 1: 49-52, 2002.

3. Wistuba II: Genetics of preneoplasia: lessons from lung cancer. Curr Mol Med 7: 3-14, 2007.

4. Miller YE: Pathogenesis of lung cancer: 100 year report. Am J Respir Cell Mol Biol 33: 216-223, 2005.

5. Lagos-Quintana M, Rauhut R, Lendeckel W and Tuschl T: Identification of novel genes coding for small expressed RNAs Science 294: 853-858, 2001.

6. Garzon R, Marcucci G and Croce CM: Targeting microRNAs in cancer: rationale, strategies and challenges. Nat Rev Drug Discov 9: 775-789, 2010

7. Eder M and Scherr M: MicroRNA and lung cancer. N Engl J Med 352: 2446-2448, 2005.

8. Landi MT, Zhao Y, Rotunno M, et al: MicroRNA expression differentiates histology and predicts survival of lung cancer. Clin Cancer Res 16: 430-441, 2010.

9. Bishop JA, Benjamin H, Cholakh H, et al: Accurate classification of non-small cell lung carcinoma using a novel microRNA-based approach. Clin Cancer Res 16: 610-619, 2010.

10. Fassina A, Cappellesso R and Fassan M: Classification of non-small cell lung carcinoma in transthoracic needle specimens using microRNA expression profiling. Chest 140: 1305-1311, 2011

11. Sassen S, Miska EA and Caldas C: MicroRNA: implications for cancer. Virchows Archiv 452: 1-10, 2008.

12. Zhang JG, Wang JJ, Zhao F, Liu Q, Jiang K and Yang GH MicroRNA-21 (miR-21) represses tumor suppressor PTEN and promotes growth and invasion in non-small cell lung cancer (NSCLC). Clin Chim Acta 411: 846-852, 2010.

13. Lu Z, Liu M, Stribinskis V, et al: MicroRNA-21 promotes cell transformation by targeting the programmed cell death 4 gene. Oncogene 27: 4373-4379, 2008.

14. Fassan M, Pizzi M, Giacomelli L, et al: PDCD4 nuclear loss inversely correlates with miR-21 levels in colon carcinogenesis. Virchows Arch 458: 413-419, 2011

15. Jiang L, Huang Q, Zhang S, et al: Hsa-miR-125a-3p and hsa-miR-125a-5p are downregulated in non-small cell lung cancer and have inverse effects on invasion and migration of lung cancer cells. BMC cancer 10: 318, 2010.

16. Arora S, Ranade AR, Tran NL, et al: MicroRNA-328 is associated with (non-small) cell lung cancer (NSCLC) brain metastasis and mediates NSCLC migration. Int J Cancer 129: 2621-2631, 2011.

17. Wang X, Ling C, Bai Y and Zhao J: MicroRNA-206 is associated with invasion and metastasis of lung cancer. Anat Rec (Hoboken) 294: 88-92, 2011.

18. Yanaihara N, Caplen N, Bowman E, et al: Unique microRNA molecular profiles in lung cancer diagnosis and prognosis. Cancer Cell 9: 189-198, 2006.

19. Volinia S, Calin GA, Liu CG, et al: A microRNA expression signature of human solid tumors defines cancer gene targets. Proc Natl Acad Sci USA 103: 2257-2261, 2006.

20. Jeong HC, Kim EK, Lee JH, et al: Aberrant expression of let-7a miRNA in the blood of non-small cell lung cancer patients. Mol Med Rep 4: 383-387, 2011.

21. Boeri M, Verri C, Conte D, et al: MicroRNA signatures in tissues and plasma predict development and prognosis of computed tomography detected lung cancer. Proc Natl Acad Sci USA 108: 3713-3718, 2011.

22. Foss KM, Sima C, Ugolini D, et al: miR-1254 and miR-574-5p: serum-based microRNA biomarkers for early-stage non-smal cell lung cancer. J Thorac Oncol 6: 482-488, 2011.

23. Liu X, Sempere LF, Ouyang H, et al: MicroRNA-31 functions as an oncogenic microRNA in mouse and human lung cancer cells by repressing specific tumor suppressors. J Clin Invest 120: 1298-1309, 2010.

24. Markou A, Sourvinou I, Vorkas PA, Yousef GM and Lianidou E: Clinical evaluation of microRNA expression profiling in non small cell lung cancer. Lung Cancer 81: 388-396, 2013.

25. Paci M, Rapicetta C and Maramotti S: New biomarkers for lung cancer. Expert Opin Med Diagn 4: 201-224, 2010.

26. Xu C, Zheng Y, Lian D, et al: Analysis of microRNA expression profile identifies novel biomarkers for non-small cell lung cancer. Tumori 101: 104-110, 2015.

27. Solomides CC, Evans BJ, Navenot JM, et al: MicroRNA profiling in lung cancer reveals new molecular markers for diagnosis. Acta Cytol 56: 645-654, 2012.
28. Guan P, Yin Z, Li X, Wu W and Zhou B: Meta-analysis of human lung cancer microRNA expression profiling studies comparing cancer tissues with normal tissues. J Exp Clin Cancer Res 31: 54, 2012.

29. Ma L, Huang Y, Zhu W, et al: An integrated analysis of miRNA and mRNA expressions in non-small cell lung cancers. PloS One 6: e26502, 2011.

30. Abrahamsen HN, Steiniche T, Nexo E, et al: Towards quantitative mRNA analysis in paraffin-embedded tissues using real-time reverse transcriptase-polymerase chain reaction: a methodological study on lymph nodes from melanoma patients. J Mol Diagn 5: 34-41, 2003.

31. Mott JL, Kobayashi S, Bronk SF and Gores GJ: mir-29 regulates Mcl-1 protein expression and apoptosis. Oncogene 26: 6133-6140, 2007.

32. Osada $\mathrm{H}$ and Takahashi T: let-7 and miR-17-92: small-sized major players in lung cancer development. Cancer Sci 102: 9-17, 2011.

33. Michael MZ, O'Connor SM, van Holst Pellekaan NG, Young GP and James RJ: Reduced accumulation of specific microRNAs in colorectal neoplasia. Mol Cancer Res 1: 882-891, 2003.

34. Zhu S, Si ML, Wu H and Mo YY: MicroRNA-21 targets the tumor suppressor gene tropomyosin 1 (TPM1). J Biol Chem 282: 14328-14336, 2007.

35. Tan X, Qin W, Zhang L, et al: A 5-microRNA signature for lung squamous cell carcinoma diagnosis and hsa-miR-31 for prognosis. Clin Cancer Res 17: 6802-6811, 2011.

36. Ciafrè SA, Galardi S, Mangiola A, et al: Extensive modulation of a set of microRNAs in primary glioblastoma. Biochem Biophys Res Commun 334: 1351-1358, 2005.

37. Cahill S, Smyth P, Finn SP, et al: Effect of ret/PTC 1 rearrangement on transcription and post-transcriptional regulation in a papillary thyroid carcinoma model. Mol cancer 5: 70, 2006.

38. Gao W, Yu Y, Cao H, et al: Deregulated expression of miR-21, miR-143 and miR-181a in non small cell lung cancer is related to clinicopathologic characteristics or patient prognosis. Biomed Pharmacother 64: 399-408, 2010.

39. Busacca S, Germano S, De Cecco L, et al: MicroRNA signature of malignant mesothelioma with potential diagnostic and prognostic implications. Am J Respir Cell Mol Biol 42: 312-319, 2010.

40. Huang JY, Cui SY, Chen YT, et al: MicroRNA-650 was a prognostic factor in human lung adenocarcinoma and confers the docetaxel chemoresistance of lung adenocarcinoma cells via regulating Bcl-2/Bax expression. PloS One 8: e72615, 2013.

41. Xiao W, Bao ZX, Zhang CY, et al: Upregulation of miR-31* is negatively associated with recurrent/newly formed oral leukoplakia. PloS One 7: e38648, 2012.

42. Costa A, Afonso J, Osório C, et al: miR-363-5p regulates endothelial cell properties and their communication with hematopoietic precursor cells. J Hematol Oncol 6: 87, 2013.

43. Xu X, Wu X, Wu S, et al: Study on miR-490-5p and miR-363 as novel biomarkers for the diagnosis of colorectal cancer. Zhonghua Wei Chang Wai Ke Za Zhi 17: 45-50, 2014 .(In Chinese).

44. Sun Q, Zhang J, Cao W, et al: Dysregulated miR-363 affects head and neck cancer invasion and metastasis by targeting podoplanin. Int J Biochem Cell Biol 45: 513-520, 2013.

45. Zhi F, Cao X, Xie X, et al: Identification of circulating microRNAs as potential biomarkers for detecting acute myeloid leukemia. PloS One 8: e56718, 2013.

46. Kelly AD, Hill KE, Correll M, et al: Next-generation sequencing and microarray-based interrogation of microRNAs from formalin-fixed, paraffin-embedded tissue: preliminary assessment of cross-platform concordance. Genomics 102: 8-14, 2013.

47. Oue N, Anami K, Schetter AJ, et al: High miR-21 expression from FFPE tissues is associated with poor survival and response to adjuvant chemotherapy in colon cancer. Int J Cancer 134: 1926-1934, 2014

48. Foss RD, Guha-Thakurta N, Conran RM and Gutman P: Effects of fixative and fixation time on the extraction and polymerase chain reaction amplification of RNA from paraffin-embedded tissue. Comparison of two housekeeping gene mRNA controls. Diagn Mol Pathol 3: 148-155, 1994

49. Doleshal M, Magotra AA, Choudhury B, et al: Evaluation and validation of total RNA extraction methods for microRNA expression analyses in formalin-fixed, paraffin-embedded tissues. J Mol Diagn 10: 203-211, 2008.

50. Jones CI, Zabolotskaya MV, King AJ, et al: Identification of circulating microRNAs as diagnostic biomarkers for use in multiple myeloma. Br J Cancer 107: 1987-1996, 2012.

51. Giray BG, Emekdas G, Tezcan S, et al: Profiles of serum microRNAs; miR-125b-5p and miR223-3p serve as novel biomarkers for HBV-positive hepatocellular carcinoma. Mol Biol Rep 41: 4513-4519, 2014. 